\title{
UTILIZAÇÃO DA TECNOLOGIA ARDUINO COMO FERRAMENTA PARA SENSORIAMENTO DE PROCESSOS DE BANCADA
}

\author{
P. FERREIRA. $\mathrm{F}^{1}$ e L.R.S. BUENO ${ }^{2}$ \\ ${ }^{1}$ UNISOCIESC - Instituto Superior Tupy, Departamento de Engenharia Química \\ ${ }^{2}$ Católica de Santa Catarina, Departamento de Química Tecnológica \\ E-mail para contato: priscillaaaferreira@hotmail.com
}

\begin{abstract}
RESUMO - Compreendendo a importância dos processos intermediários em termos de escalas, normalmente carentes de automação devido a elevados custos, propõe-se a utilização da ferramenta Arduino para controle e monitoramento de processos pilotos e laboratoriais. A confiabilidade, viabilidade e principalmente a capacidade de aquisição de dados desta ferramenta foi verificada por meio de comparação da curva de pressão de vapor (superfície P-T) do procedimento manual, automatizado pelo Arduino e quando comparados aos dados do programa Engineering Equation Solver (EES), (adotado como referência). Os resultados se mostraram satisfatórios, pois para o procedimento manual obteve-se um desvio de $52 \%$ em relação ao EES, enquanto que para o automatizado apenas $8 \%$ de diferença, sendo que ambos foram realizados nas mesmas condições operacionais. Este trabalho permite uma interdisciplinaridade dos assuntos tratados em engenharia e abre novos horizontes para automação, a baixos custos, nos mais variados processos.
\end{abstract}

\section{INTRODUÇÃO}

A tendência em automatizar processos vem crescendo de forma expressiva nos diversos segmentos de engenharia. Com o surgimento do microcontrolador Arduino, em 2005, esta tarefa de sensoriar e monitorar com confiabilidade tornou-se acessível para diferentes profissionais. Embora exista uma distância considerável entre escala laboratorial e a produção industrial, sendo suprido pela escala piloto devido ao aumento de quantidade de matéria envolvida, o controle do processo intermediário normalmente é carente de automação. Tais atributos são frequentemente encontrados devido aos elevados custos de equipamentos para efetiva automação do processo.

A possibilidade de aplicação ampla deste novo artifício em processo intermediários, assim como experimentos laboratoriais, é resultante da facilidade de comunicação pela interface homem máquina, propiciada pela linguagem computacional $\mathrm{C}++$. Para validação do Arduino como ferramenta para monitoramento de processos químicos, este estudo almeja realizar um comparativo entre dados obtidos por procedimento experimental com e sem a utilização do microcontrolador Arduino, tomando como base comparativa os dados adquiridos pelo software Engineering Equation Solver-EES. 
Com base na termodinâmica aplicada, propôs-se uma metodologia para obtenção da curva de pressão de vapor de etanol puro, sendo escolhido pela importância deste fenômeno nas indústrias e processos químicos. A pressão de vapor pode ser medida por vários métodos, sendo a mais conhecida a metodologia Reid. Hans (2013), afirma que todos esses métodos demandam tempo, equipamentos sofisticados, pessoal técnico especializado, e custos elevados.

Apresentando-se como uma alternativa rápida e de baixo custo, a utilização da ferramenta Arduino serve como prototipagem para controle de processos químicos e mecânicos, contribuindo também para a produção de metodologia para interdisciplinaridade dos conteúdos apresentados no transcorrer do curso de engenharia.

\section{REVISÃO BIBLIOGRÁFICA}

\subsection{Automação - Microcontrolador Arduino}

As condições internas e externas que afetam o desempenho de um processo, como temperatura, pressão, nível, vazão e volume, são chamadas variáveis de processo - sendo estas, sujeitas a sistemas de controle atuantes pela intervenção humana ou artifícios automáticos - a automação. Para realizar o controle e sensoriamento destas variáveis de processos sejam eles aplicados em escalas laboratoriais ou sistemas intermediários propõem-se uma ferramenta alternativa, os micro controladores (MARCO \& LEANDRO, 2011).

Projetada com um micro controlador Atmel AVR de placa única, o Arduino é uma plataforma de prototipagem eletrônica de hardware livre com suporte de entrada/saída embutido. Desde que o Arduino Project teve início em 2005, mais de 150.000 placas oficiais de Arduino foram vendidas em todo o mundo em seis anos. A descoberta desta ferramenta está se difundindo rapidamente e umas das vantagens apresentadas por este artifício é a possibilidade de criar projetos interessantes, rápidos e fáceis, com uma curva de aprendizado relativamente pequena. McRoberts (2012) menciona que utilizando um micro controlador torna-se possível integração com sensores, motores e outros dispositivos eletrônicos. Existe uma vasta gama de sensores analógicos e digitais capazes de fazer a comunicação com o Arduino, variando desde sensores de temperatura, pressão, umidade, identificador de álcool em estado vapor, assim como sensor de presença, ultrasônico, sensor de voz, rotação, distância, entre outros.

\subsection{Mudanças de Fases e Pressão de Vapor}

O conceito de equilíbrio, termo fundamental para compreensão de processos químicos, é caracterizado por uma condição de estabilidade mantida por uma igualdade de forças que se opõem. Quando um sistema está isolado, seu estado pode mudar como uma consequência de eventos espontâneos que estejam ocorrendo internamente à medida que suas propriedades intensivas, como temperatura ou a pressão apresentam valores uniformes (SONNTAG e BORGNAKKE, 2011). A partir de dados experimentais, Shapiro e Moran (2009) afirmam que a temperatura e o volume específico podem ser considerados independentes e a pressão determinado como função destes dois fenômenos. 
A Figura 1, corresponde a uma substância que se contrai durante a solidificação, sendo que a maioria das substâncias exibe este comportamento. As coordenadas de um ponto na superfície P-V-T representam os valores que a pressão, o volume específico e a temperatura assumem quando a substância se encontra no equilíbrio.

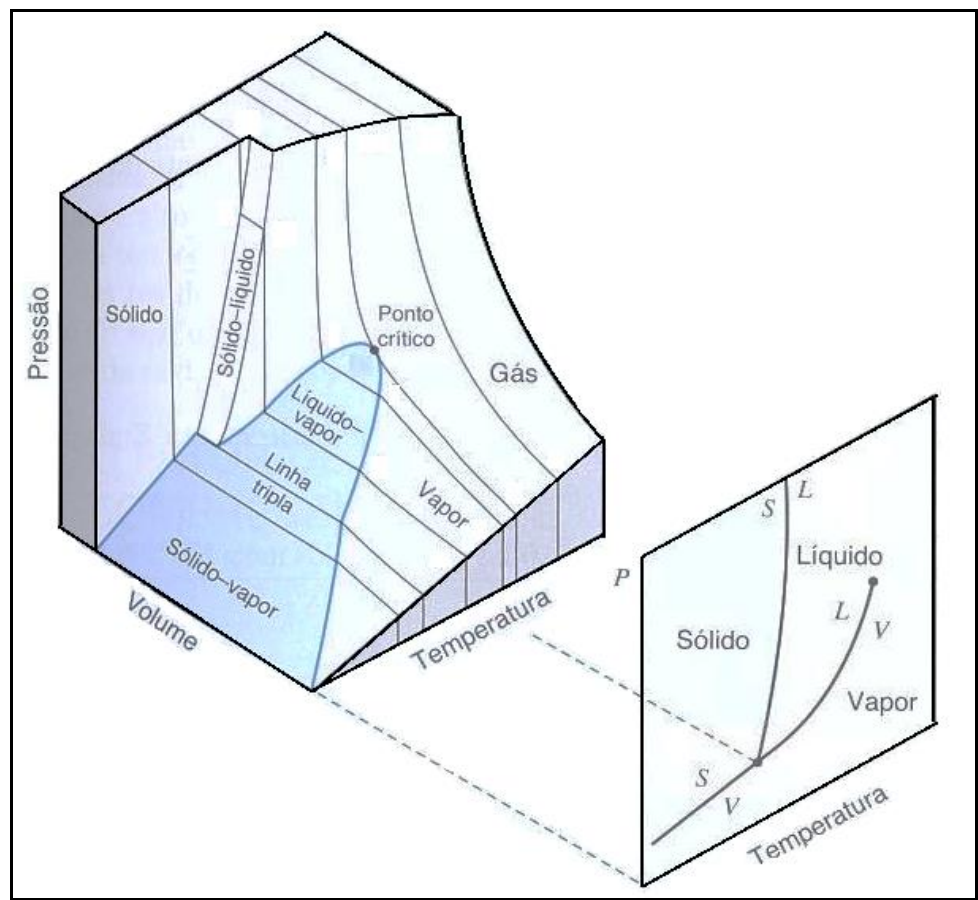

Figura 1 - (a) Superfície P-V-T.

A superfície P-V-T (Pressão - Volume - Temperatura) é útil para se enfatizar as relações gerais entre as três fases da matéria que são geralmente consideradas, entretanto, a utilização de projeções bidimensionais dessa superfície são geralmente mais convenientes. Se a superfície P-V-T for projetada sobre o plano pressão versus temperatura obtêm-se um diagrama de propriedades conhecido como diagrama de fases (SONNTAG e BORGNAKKE, 2006). Um termo termodinâmico que se destaca perante este estudo é a temperatura de saturação, que é assim denominada por mostrar a temperatura na qual uma mudança de fase ocorre para uma dada pressão de saturação.

A pressão de vapor é uma das propriedades físicas mais importantes em combustíveis e também é uma variável considerada em processos químicos que apresentam variação na pressão e temperatura. Conforme cita Cruz (2003), nestas técnicas de obtenção da curva de pressão de vapor, a câmara do líquido do equipamento medidor de pressão de vapor é preenchida com a amostra resfriada e conectada à câmara do vapor que é aquecida a uma dada temperatura específica para cada substância. A relação volumétrica entre a câmara de vapor e a câmara líquida é de 4:1. A pressão medida através destes procedimentos é definida como a pressão de vapor Reid (RVP) (ASTM, 1993).

\section{PROCEDIMENTO EXPERIMENTAL}

O processo a ser monitorado é a obtenção da curva de pressão de vapor para o fluido Etanol 
PA. Os dados coletados serão comparados com os dados obtidos pelas curvas gerados pelo programa computacional EES e também com o mesmo procedimento medido manualmente.

Para ambos os procedimentos, manual e automatizado, liga-se a bomba a uma determinada pressão, após a estabilização da mesma, adiciona-se calor ao sistema através da manta de aquecimento. Para cada pressão, haverá um instante em que a temperatura permanecerá brevemente constante, neste momento anota-se os valores correspondentes à pressão de saturação e temperatura de saturação. No procedimento manual, a leitura da pressão realiza-se diretamente no manômetro da bomba e para a temperatura utiliza-se o termômetro digital. No procedimento automatizado, utiliza-se o arduino como ferramenta para aquisição de dados, sendo que para medição de temperatura usa-se o Termistor $10 \mathrm{~K} \Omega$ (Marca HUADY) e para medir a pressão o sensor MPX5700dP (Marca FRESSCALE). Neste sistema, não é necessário fazer a leitura manual dos valores de saturação, pois os sinais durante o experimento são diretamente armazenados no computador, Figura 2.

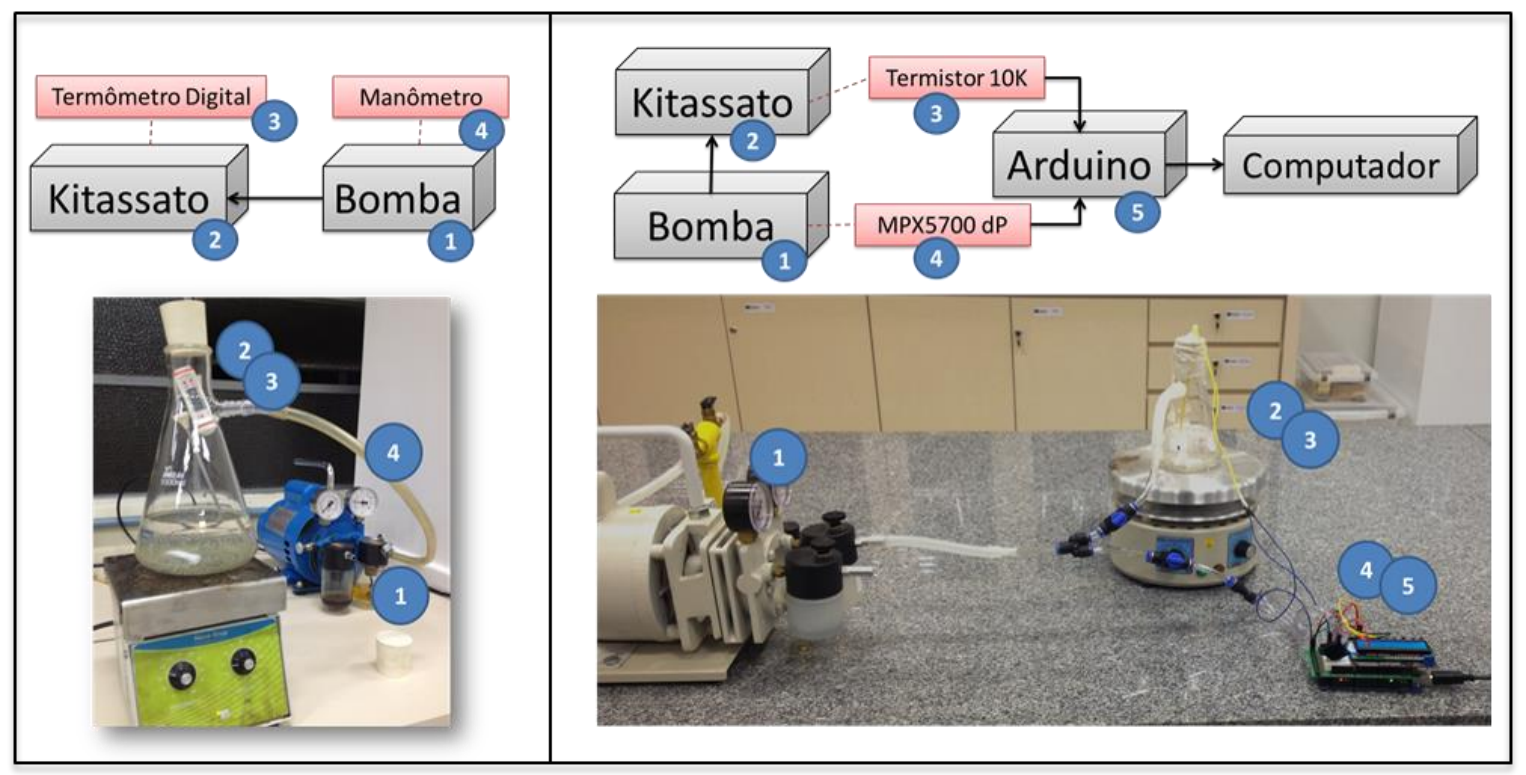

Figura 2 - Procedimento manual (esquerda) e automatizado (direita).

Diante das variáveis presentes no procedimento experimental, torna-se necessário fazer algumas considerações: a) Admite-se que a temperatura seja alterada a uma taxa de elevação constante, não ocorrendo influências externas que alteram esta ação; b) Não existe entrada de ar ocasionando algum vazamento no sistema e consequentemente instabilizando a pressão;

\section{ANÁLISE DE DADOS}

Todos os dados de temperatura e pressão do procedimento automatizado foram coletados e organizados conforme a Figura 3 e Figura 4, respectivamente. 


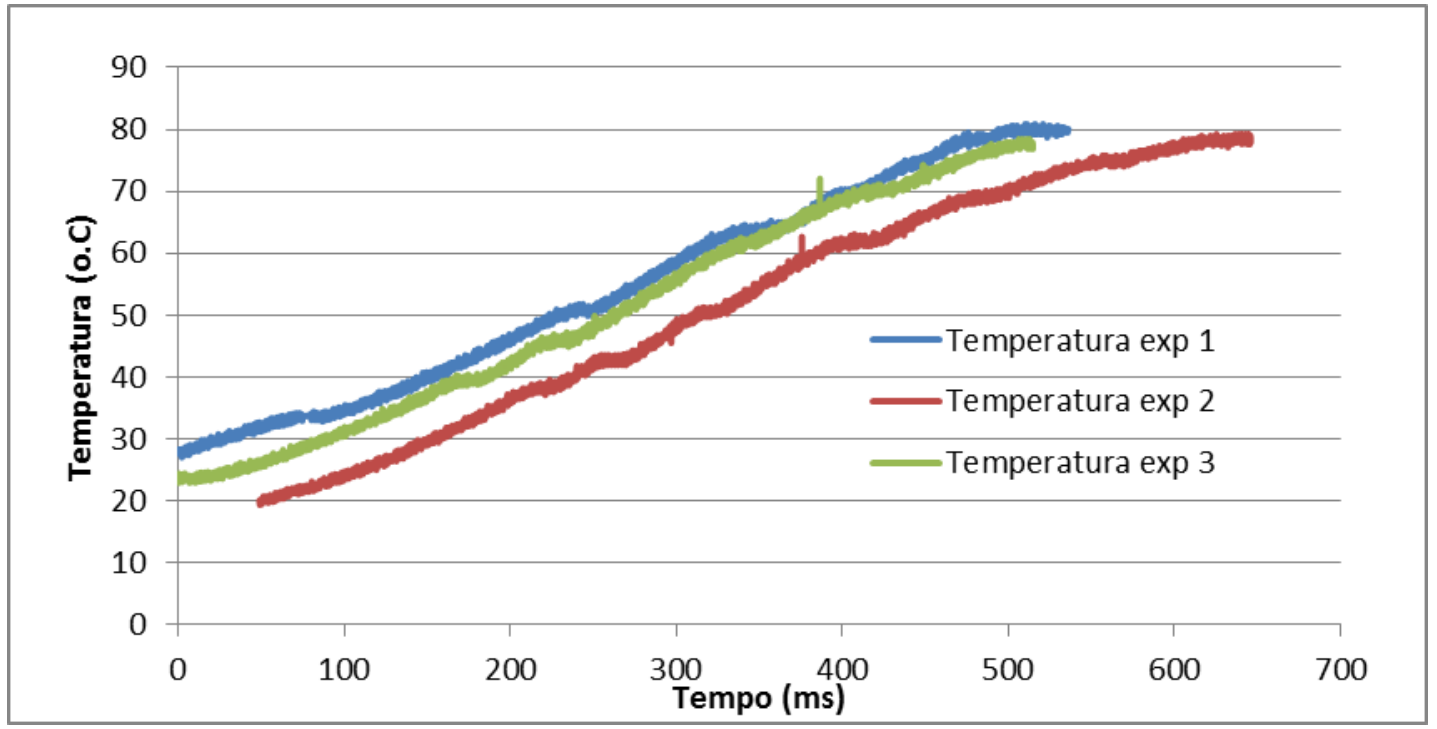

Figura 3 - Dados de Experimentais de Temperatura.

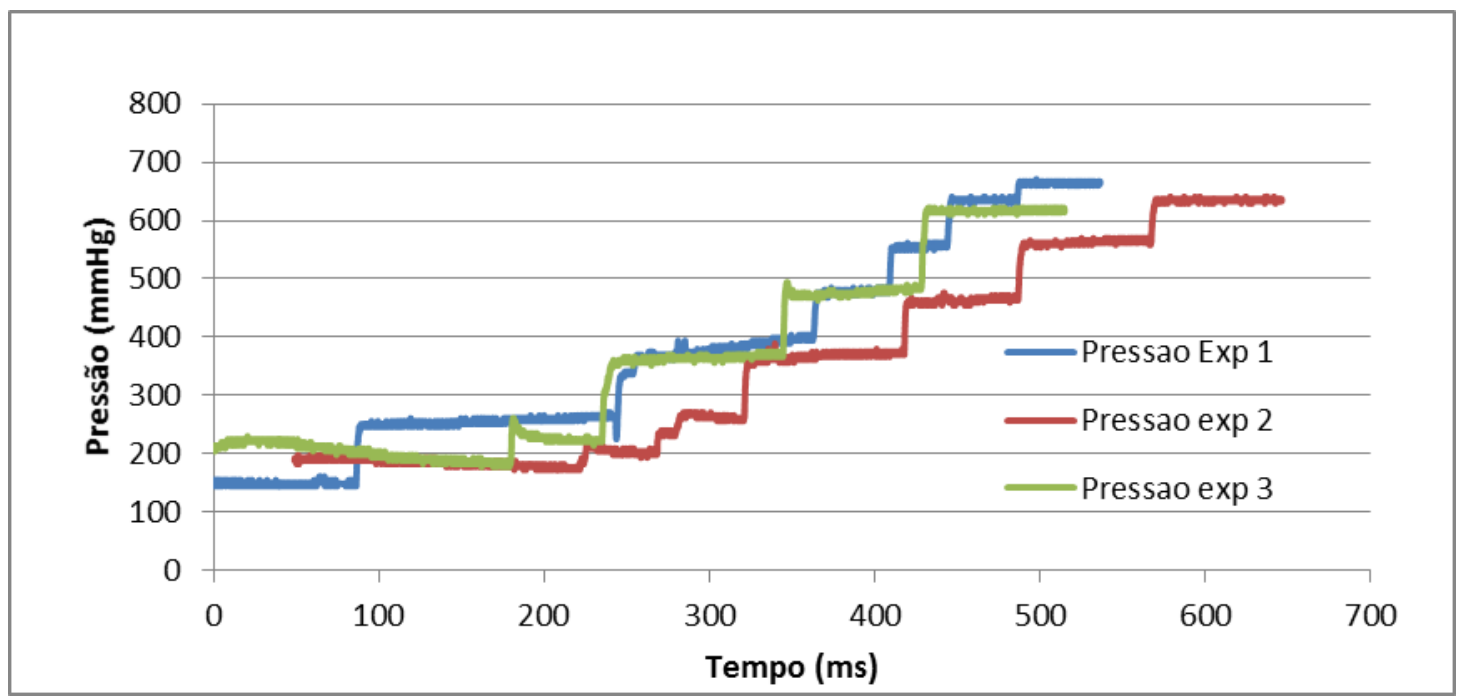

Figura 4 - Dados Experimentais de Pressão.

Os degraus presentes na Figura 4, representam a mudança de pressão realizada manualmente, por este motivo ocorreu uma divergência entre as curvas dos três experimentos apresentados, no entanto como a curva de pressão de vapor é determinada pelo equilíbrio entre temperatura e pressão para cada experimento, esta diferença apenas fornecerá novos pontos para a mesma curva. Observando com mais ênfase o Experimento 1, Figura 5 e Figura 6 nota-se alguns pontos constantes. 


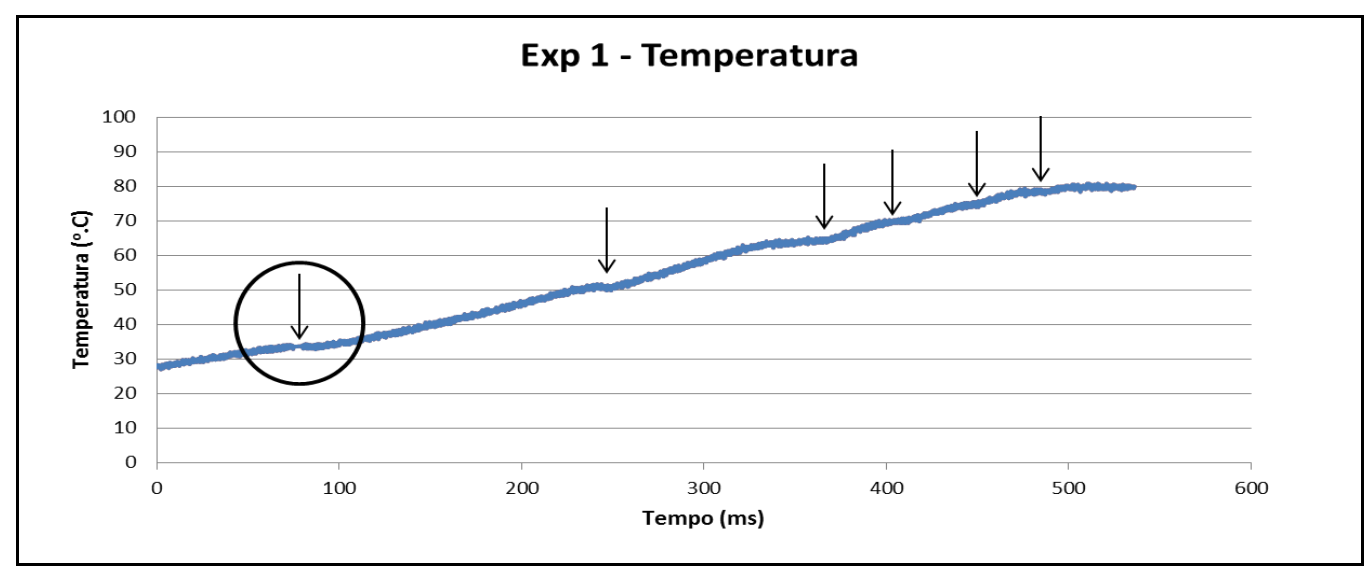

Figura 5 - Dados de Temperatura para Experimento 1.

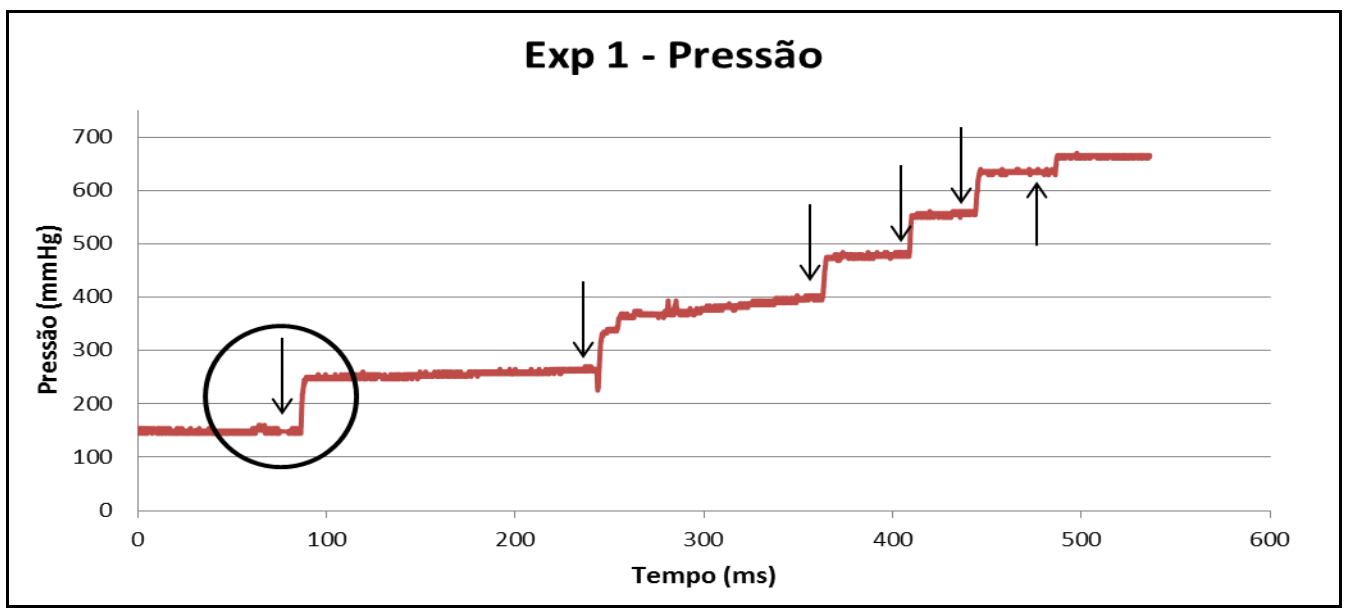

Figura 6 - Dados de Pressão para Experimento 1.

É notável a estabilização da curva em ambos gráficos para o mesmo instante, ponto importante para a determinação da superfície P-T da substância analisada. Quanto se agrupa os valores de saturação obtêm-se o gráfico de superfície P-T, este que demostra a curva de vapor do líquido, que neste estudo é o álcool etílico PA.

Na Figura 7, apresenta-se a curva do experimento 1, observando com mais aproximação o comportamento da curva geradas pelo procedimento automatizado, no momento da saturação. Onde Ambas curvas, pressão e temperatura, se mantem constante. 


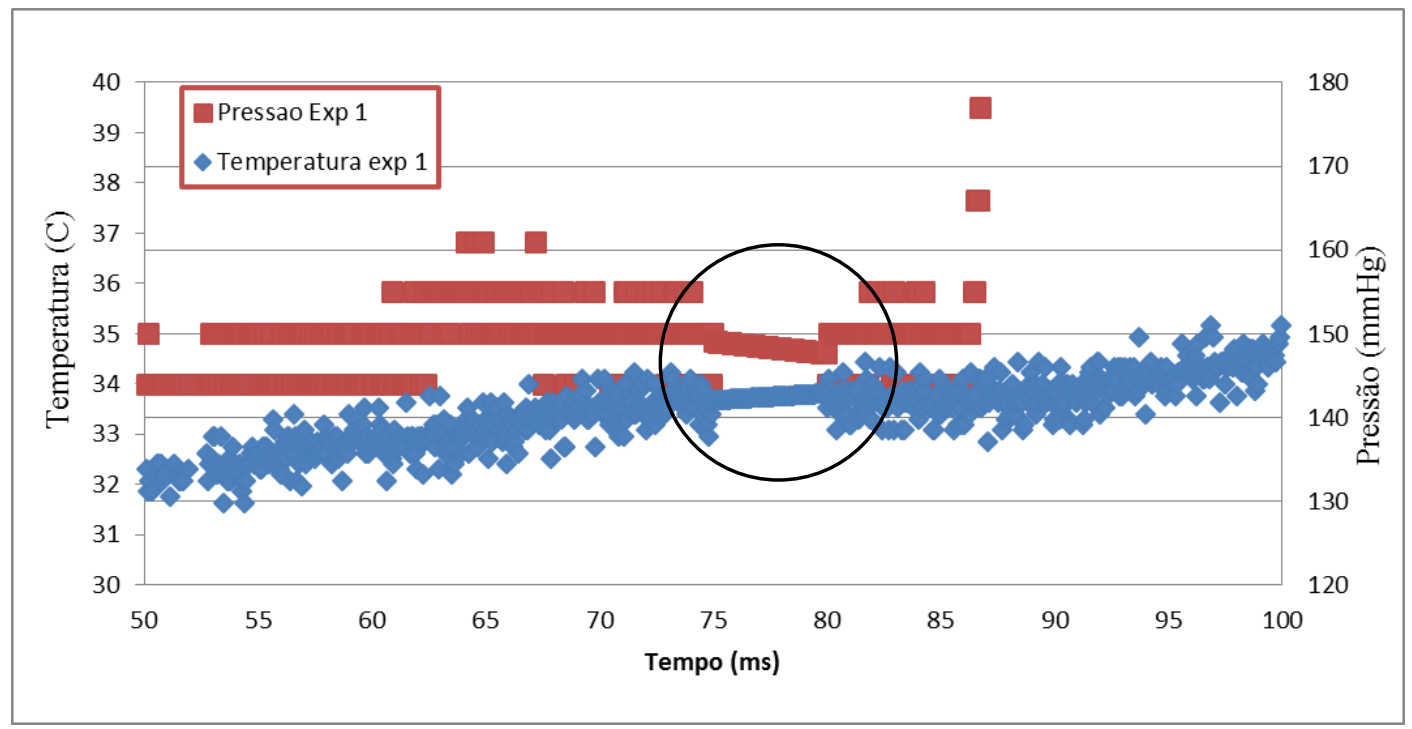

Figura 7 - Dados de Pressão e Temperatura para Experimento 1.

Na Figura 8, apresenta-se a curva dos experimentos 1, 2 e 3, do programa computacional EES e também do procedimento manual.

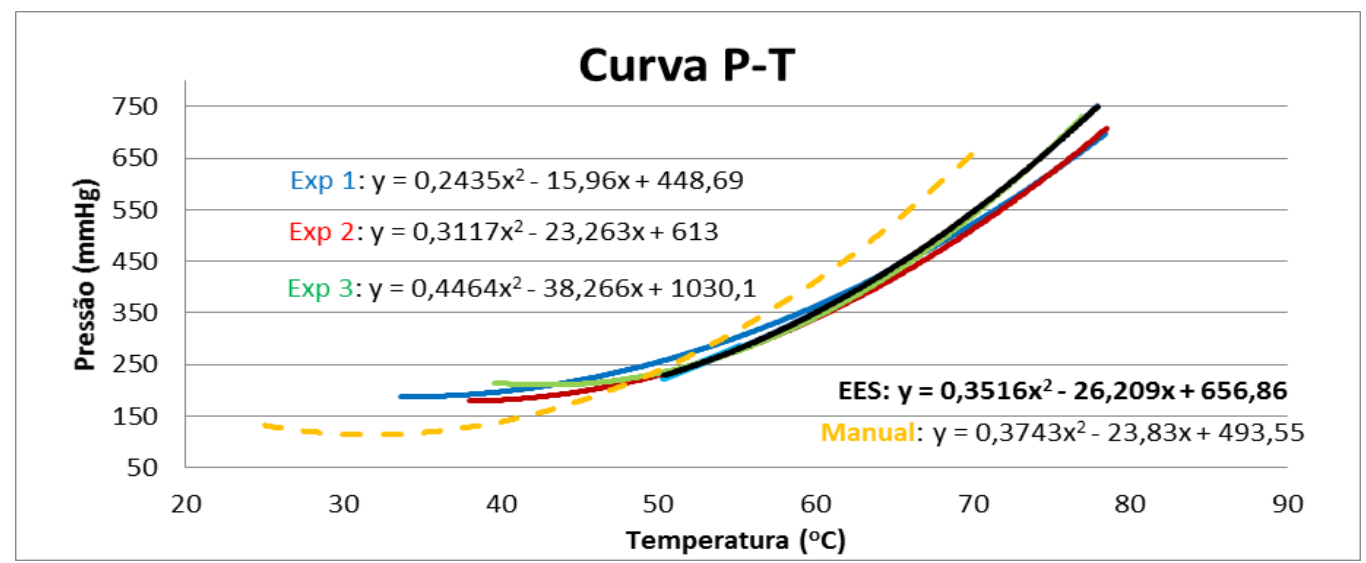

Figura 7 - Comparativo de Curva P-T.

A curva de pressão de vapor ou superfície P-T definida pelo EEs na cor preta é a base comparativa. Pode-se verificar a semelhança para os experimentos 1,2 e 3, e também a distância da curva do procedimento manual em Laranja. A diferença apresentada provém da dificuldade de coletar dados no procedimento manual. Como a mudança de fase ocorre muito rapidamente, cerca de dez mili segundos, qualquer fração de tempo faz alterar consideravelmente a curva de pressão de vapor. 


\section{CONCLUSÃO}

A utilização da ferramenta Arduino foi designada para criar dispositivos para controlar e monitorar projetos protótipos de modo menos dispendioso. Sendo necessário ter conhecimento de linguagem computacional $\mathrm{C}$, e para o usuário conhecer os fundamentos de eletrônica, este estudo comprova que esta ferramenta pode sim se tornar um utensílio confiável para monitorar processos químicos. Sejam eles laboratoriais ou pilotos, desde que estejam dentro da faixa de leitura e especificações de cada dispositivo.

Para as mesmas condições de procedimento experimental, a curva de pressão de vapor para a coletada de dados manual, quando comparada aos dados do EES, foi de aproximadamente $52 \%$ de desvio, enquanto que para o procedimento utilizando o Arduino foi de apenas $8 \%$ de diferença. A precisão e a capacidade de aquisição de dados oferecida pelo Arduino e seus sensores possibilitam uma aproximação das curvas geradas, pois ocorre cerca de trinta leituras por segundo. Enquanto que no procedimento sem automação coleta-se cerca de vinte dados por experimento devido à rapidez de troca de temperatura e pressão de saturação do sistema, o delay da pessoa que acompanha o procedimento e confiabilidade da coleta que é via manômetro analógico e termômetro digital.

A aplicação desta ferramenta se difunde rapidamente e umas das vantagens confirmadas neste estudo é a facilidade de comunicação com a interface homem máquina. A linguagem é amplamente alastrada nos meios acadêmicos, e por ser uma plataforma aberta existe mútua troca de informação proporcionando mais interação e possibilidade de criação de novos projetos. Seu baixo custo permite facilidade de acesso e pela variedade de sensores existentes.

\section{REFERENCIAS}

ASTM, American Society for Testing and Material, ASTM Committee of Standards, West Conshohocken; Standard Test Method for Vapor Pressure of Gasoline and Gasoline-Oxigenate Blends - Dry Method; ASTM D 4953, 1993.

CRUZ, M. J. F. Determinação Experimental e Predição da Pressão de Vapor de Gasolinas com Aditivos Oxigenados. Dissertação apresentada ao Curso de Mestrado (Engenharia Química) da Universidade Federal de Pernambuco, 2003.

MARCO J.L.G., LEANDRO G.V. MICROCONTROLADOR PIC 18F452 / PROTEUS. Universidade Federal Do Paraná. Curitiba, 2011.

McRoberts M. Arduino Básico. Novatec Editora, São Paulo, 2012.

SHAPIRO, H. N., MORAN, M.1 J. Princípios De Termodinâmica Para Engenharia. LTC, Ed.6; Rio de Janeiro, RJ, 2009.

SONNTAG, R. E., BORGNAKKE, C. Introdução à Termodinâmica para Engenharia. 2a edição, Editora Koury, Rio de Janeira, 2011. 
HAN S. P., YOUN I. M., LIM Y. LEE C. C. Influence of the mixture of gasoline and diesel fuels on droplet atomization, combustion, and exhaust emission characteristics in a compression ignition engine. School of Mechanical Engineering, Hanyang University, Republic of Korea, Fuel Processing Technology 106, N. 392-401., 2013. 